\title{
CFD Study of Liquid Sodium inside a Wavy Tube for Laminar Convectors: Effect of Reynolds Number, Wave Pitch, and Wave Amplitude
}

\author{
Syed Murtuza Mehdi, ${ }^{1}$ Maaz Akhtar, ${ }^{1}$ Ahmad Hussain, ${ }^{1}$ \\ Dheya Shuja Alothmany, ${ }^{2}$ and Shahid Aziz ${ }^{3}$ \\ ${ }^{1}$ Department of Mechanical Engineering, NED University of Engineering \& Technology, Karachi, Pakistan \\ ${ }^{2}$ Department of Nuclear Engineering, King Abdulaziz University, Jeddah, Saudi Arabia \\ ${ }^{3}$ Department of Mechatronics Engineering, Jeju National University, Jeju, Republic of Korea \\ Correspondence should be addressed to Ahmad Hussain; ahmadutm@gmail.com
}

Received 8 May 2016; Revised 25 September 2016; Accepted 12 October 2016

Academic Editor: Efstratios Tzirtzilakis

Copyright ( $) 2016$ Syed Murtuza Mehdi et al. This is an open access article distributed under the Creative Commons Attribution License, which permits unrestricted use, distribution, and reproduction in any medium, provided the original work is properly cited.

\begin{abstract}
Metallic tubes have been widely used as primary heat transfer elements in laminar convectors for domestic and aerospace heating purpose. This paper uses CFD tool to investigate the heat output and pressure drop of liquid sodium flowing inside a circular tube having a wavy profile throughout its length. The wavy tube can be utilized in laminar liquid metal convectors as basic heat transfer element. The effect of Reynolds number $(500 \leq \mathrm{Re} \leq 2000)$ wave pitch $(25 \mathrm{~mm} \leq \lambda \leq 100 \mathrm{~mm})$ and wave amplitude $(2 \mathrm{~mm} \leq a \leq 6 \mathrm{~mm})$ on the heat output and pressure drop has been numerically studied. Based on the CFD results important controlling parameters have been identified and it is concluded that the heat output from the wavy tube is affected by the wave pitch and the wave amplitude while the pressure drop is mostly affected by the Reynolds number and wave amplitude.
\end{abstract}

\section{Introduction}

Laminar flows involve several attractive features like low thermodynamic and hydrodynamic irreversibility, silent and vibration-free operation and longer life and greater reliability, Doty et al. [1]. Another important feature of laminar flows is that the equations of motion (i.e., the Navier-Stokes equations for fluid dynamics) do not involve any time averaging technique like the turbulent flows, which ultimately makes the numerical results pertaining to this class of fluid motion directly and more reliably applicable to engineering designs. For instance, Kays [2] numerically studied the heat transfer in laminar flows inside circular tube, Ko [3] numerically studied the entropy generation and optimum Reynolds number for laminar developing flow inside double sine ducts, Chen and Chiou [4] studied the heat transfer in the entrance region of a laminar liquid metal pipe flow, Sahin and Mansour [5] numerically studied entropy generation in laminar fluid flow inside a circular pipe, Mehdi and Choi [6] performed
CFD study of liquid sodium within thermally developing region under laminar flow, Gedik et al. [7] utilized CFD to study the laminar magnetohydrodynamics flow of liquid metal inside a circular tube, and Makinde and Eegunjobi [8] numerically studied the effects of convective heating on entropy generation for laminar flow inside a channel with permeable walls. It is important to mention that in laminar flows the convective effects are low which places limit on heat and mass transport; however, the transport of heat in laminar flows can be augmented by utilizing several alternatives which includes the use of low Prandtl number fluids such as liquid metals [6], the use of nanofluids as indicated by the work of Yang et al. [9], and increase in heat transfer area as indicated by the work of Kim et al. [10].

Consequently laminar flows can become useful for domestic and aerospace heating applications. In fact, there exist many interesting heat exchanger design concepts that are based on laminar fluid flow; for instance, Stignor [11] presented the concept of laminar flat tube heat exchangers; 
Miner and Ghoshal [12] studied liquid metals as coolant for laminar microcoolers; similarly Tawk et al. [13] investigated different design parameters for microcoolers to be exploited in electronic cooling; Lin et al. [14] investigated the performance of a laminar heat pipe heat exchanger; Iqbal and Syed [15] presented the concept of laminar finned doublepipe heat exchanger; Saji et al. [16] developed a compact laminar microtube heat exchanger; Sagnik and Saha [17] presented the concept of utilizing simultaneously transverse corrugations and twisted tape inserts in a circular tube for augmenting the convective laminar heat transfer, whereas Sui et al. [18] investigated laminar flow and heat transfer in wavy microchannels.

Based on the extensive use of laminar flows in different types of heat exchangers, here we present the concept of wavy tube as basic heat transfer element for laminar convectors which can be used in domestic and aerospace heating applications. In this paper computational fluid dynamics approach has been used to investigate the performance (heat output and pressure drop) of the wavy tube operating under fixed set of convective boundary condition for ambient air. The length of the tube is such that the flow always remains hydrodynamically developing. Also in this study liquid sodium is used as a heat transfer fluid since liquid metals due to their high molecular heat conduction can enhance the heat transport in laminar flows.

\section{Fabrication of Wavy Tube}

A possible fabrication technique for the wavy tube is depicted schematically in Figures $1(\mathrm{a})-1(\mathrm{~d})$. The process starts with splitting a tube of desired diameter in longitudinal direction. One half of the tube is then clamped within a set of die that is designed on the basis of desired wave parameters (wave pitch and amplitude). With the application of external force the desired profile can be impregnated on the tube. The other half of the tube is then processed in similar manner and finally the two halves can be joined together by using a suitable joining process such as fusion welding. The geometry of the tube with different waviness profile (i.e., different values of wave pitch and amplitude) was built using the readymade geometry features available in the graphic user interface (GUI) of the COMSOL Multiphysics software. Thus the different wavy profiles were generated in a way that the flow radius increases and decreases by an amount equal to the wave amplitude on either side of the base radius. The value of the base radius was kept constant at $13 \mathrm{~mm}$. The shape of the waves remains like a portion of a smooth circular arc as seen in Figure 1(d).

\section{Problem Formulation}

Steady hydrodynamically developing laminar flow of liquid sodium was studied inside a wavy tube for different values of $\lambda$ and $a$ (see Figure $1(\mathrm{~d})$ ). The diameter of the tube was kept constant at $26 \mathrm{~mm}$ with an axial length of $0.55 \mathrm{~m}$. The simulated Reynolds number range was 500 to 2000 . It is well known that for steady laminar flows the hydrodynamic developing length can be estimated by the equation $\left(L_{h} \approx 20 \operatorname{Re} D\right)$, where $L_{h}$ is the hydrodynamic developing length of the flow, $D$ is the diameter of the tube, and Re is the Reynolds number [19]. According to the above equation the developing length of the problem under consideration remains larger than the length of the tube considered in this study which means that the flow always remains hydrodynamically developing. Since the geometry under consideration is axisymmetric, the governing equations, that is, (1)-(3), were numerically solved for half of the computational domain using a finite element commercial code COMSOL Multiphysics.

$$
\begin{aligned}
& \rho(\vec{V} \cdot \nabla V) \vec{V} \\
& =\nabla \cdot\left[-p I+\mu\left(\nabla \vec{V}+(\nabla \vec{V})^{t}\right)\right]-\frac{2}{3} \mu[(\nabla \cdot \vec{V}) I] \\
& +F \text {, } \\
& \nabla \cdot \vec{V}=0 \\
& \rho C \vec{V} \cdot \nabla T-\nabla \cdot(k \nabla T)=0 .
\end{aligned}
$$

Equation (1) is the famous Navier-Stokes equation for fluid motion which solves for the velocity and pressure field within the flow domain, (2) is the continuity equation (conservation of mass) for an incompressible fluid, and lastly (3) is the energy equation used to calculate the temperature field. In (1) $\rho$ is the fluid density, $\vec{V}$ is the velocity vector (composed of the axial and radial velocity components), $\nabla$ is the del operator of vector calculus, $p$ is the pressure of the fluid, $I$ is the identity matrix, $\mu$ is the dynamic viscosity of the fluid, $t$ indicates transpose of the matrix, and $F$ is the body force such as gravity. Also in (3) $C, T$, and $k$ represent the specific heat, temperature, and thermal conductivity of the working fluid, respectively. The different thermophysical properties of liquid sodium were estimated at the inlet temperature $(T)$ in Kelvin from the following [20]:

$$
\begin{aligned}
\rho= & 1014-0.235 T \\
C= & \frac{-3.001 \times 10^{6}}{T^{2}}+1658-0.8479 T+4.454 \\
& \times 10^{-4} T^{2}, \\
k= & 104-0.047 T, \\
\ln (\mu)= & \frac{556.835}{T}-0.3958 \ln (T)-6.4406 .
\end{aligned}
$$

After obtaining the complete solution (i.e., the velocity, pressure, and temperature field) the heat output and the pressure drop were calculated by using (5) and (6), respectively.

$$
\begin{aligned}
Q_{w} & =\int_{s}-\left.k \frac{d T}{d r}\right|_{\mathrm{wall}}, \\
\Delta p_{w} & =\frac{\int_{0}^{R} 2 \pi r p d r}{\int_{0}^{R} 2 \pi r d r} .
\end{aligned}
$$

In (5) $Q_{w}$ represents the heat output from the wavy tube and is calculated as the surface integral of the radial conductive 


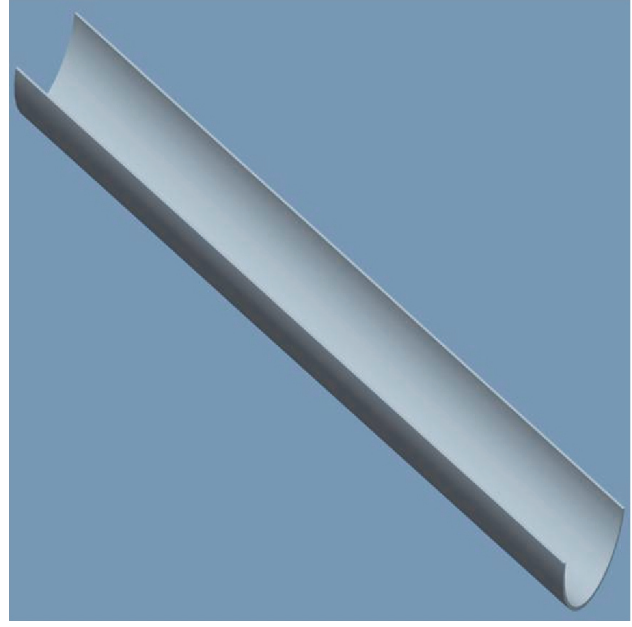

(a)

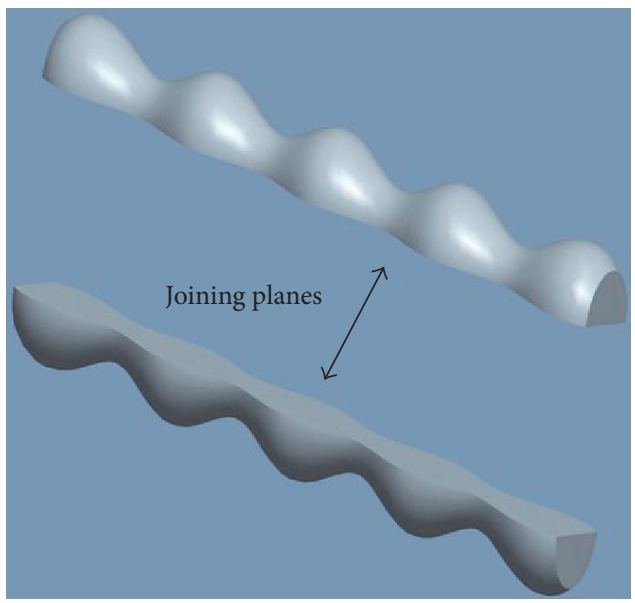

(c)

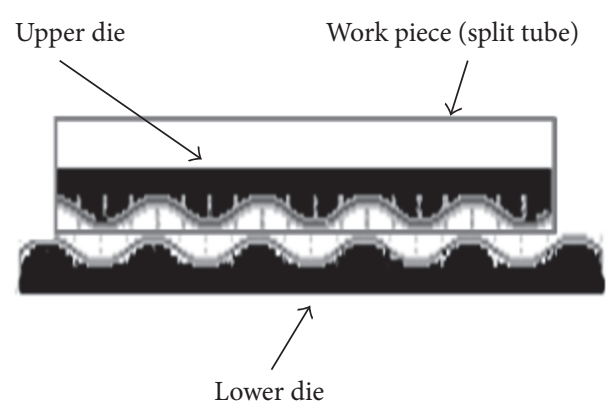

(b)

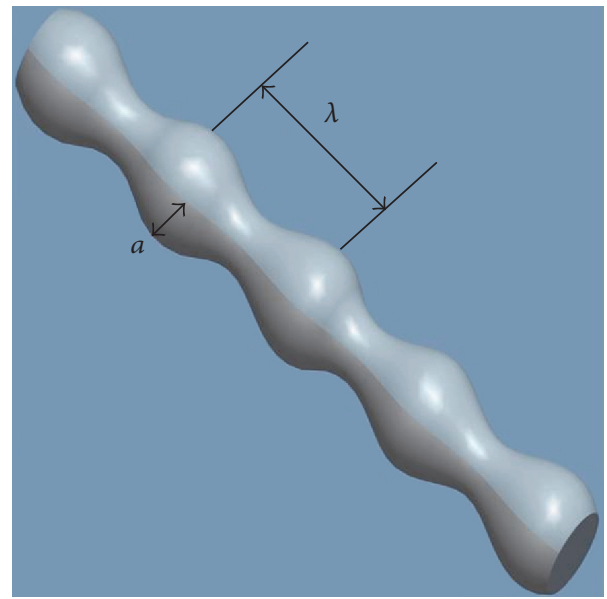

(d)

FIGURE 1: Schematic diagram depicting the conceptual steps for the fabrication of wavy tube. (a) Axially split tube of desired diameter. (b) Impregnating the desired wave length $(\lambda)$ and wave amplitude $(a)$ using a die. (c) Joining of the two parts and (d) final tube with desired wave length $(\lambda)$ and wave amplitude $(a)$.

heat flux at the wall where $r$ is the radial coordinate. Also in (6) $\Delta p_{w}$ represents the pressure drop along the wavy tube and $p$ is the pressure distribution at the inlet of the tube.

\section{Computational Details}

COMSOL Multiphysics was used to solve the governing partial differential equations, that is, (1)-(3), within the computational domain. It is to be noted that the present problem is a coupled multiphysics fluid flow problem; therefore, direct PARDISO solver was used which treats the problem as coupled and highly nonlinear. The convergence criterion for all solution variables was set to $10^{-5}$. Both the stream line and cross wind stabilization techniques were used as these stabilization techniques are important for finite element based fluid dynamics simulations. The cross wind stabilization parameter was set to 0.1 . Second-order Lagrange elements were used for the velocity and temperature variables while linear Lagrange elements were used for the pressure variable. The Lagrange elements approximate the solution variables with a piecewise polynomial function on each element of the grid. Further information on the finite element methods can be retrieved from $[21,22]$.

For the present study, the $R / 38$ (where $R$ is the radius of the tube) criterion for laminar flows was used to design the grid [23]. The $R / 38$ criterion states that the height of the wall adjacent element must be less than or equal to the ratio $R / 38$. However, in order to properly resolve the boundary layer, approximately 5 elements were embedded within a small height of $R / 38$ adjacent to the tube wall. The part of the grid near the wall is shown in Figure 2(a) which shows that presence of 5 elements within a height of $0.342 \mathrm{~mm}$, while Figure 2(b) shows the overall and the magnified view of the grid used for the simulations. It can be noticed from Figure 2(b) that structured quadrilateral grid was used with grid density being larger near the solid wall. The grid independency of the final results (i.e., the heat output and the pressure drop) was confirmed by running 


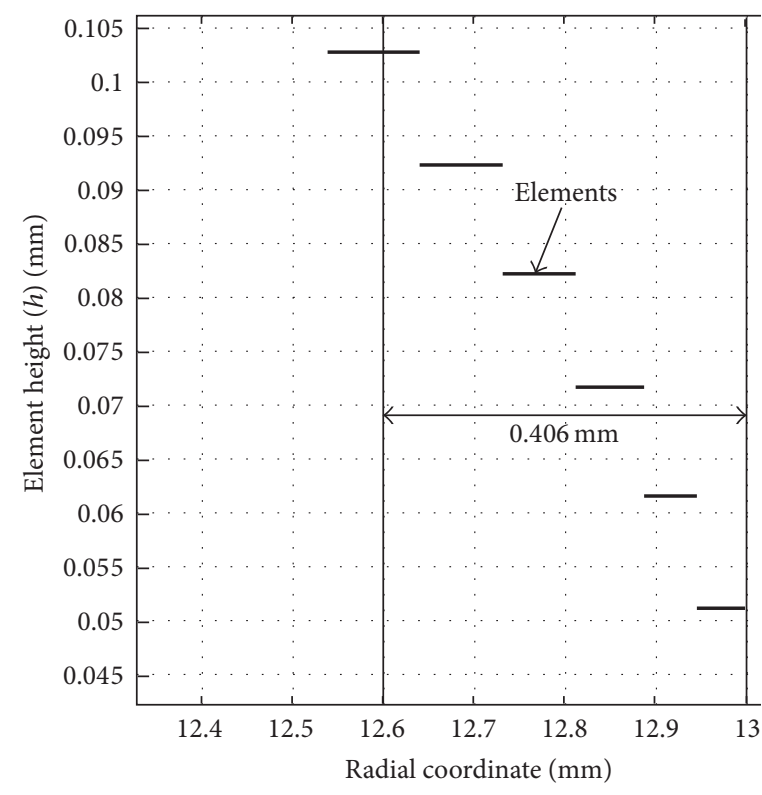

(a)

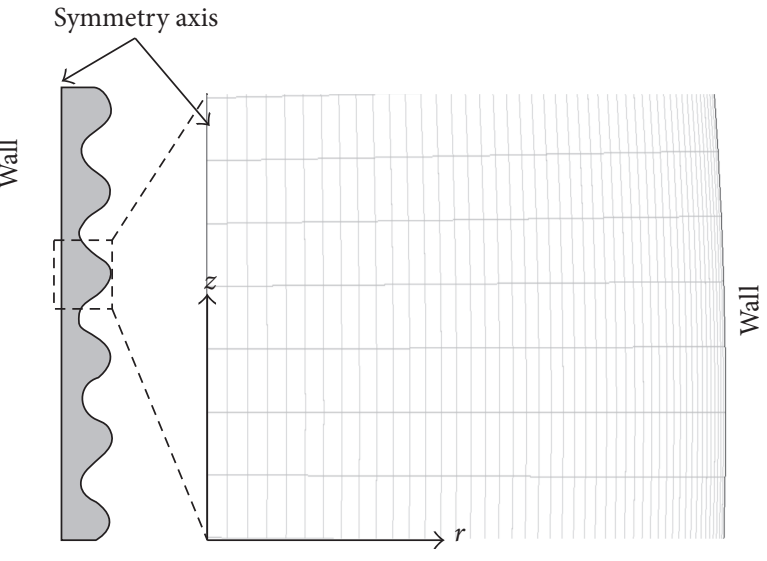

(b)

FIGURE 2: CFD grid used for the simulations. (a) Number of elements and their height near the wall within a distance of $R / 38$. (b) Overall mesh and the enlarged view of the grid showing the distribution of the elements in the domain.

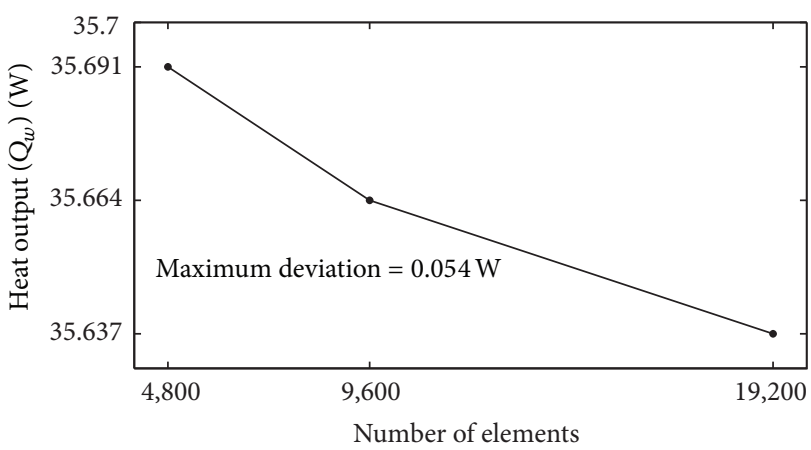

(a)

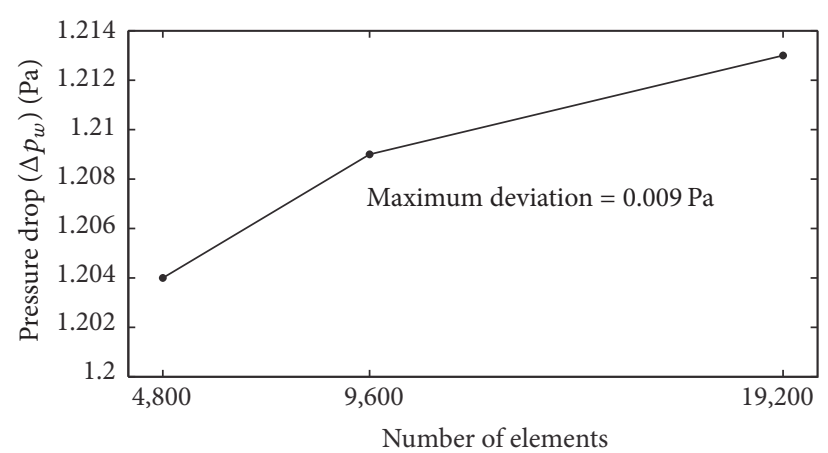

(b)

FIGURE 3: Grid independent results for (a) the heat output and (b) pressure drop.

the simulations at three different grid densities $(4800,9600$, and 19200 elements). Figures 3(a) and 3(b) indicate that no substantial deviation exists between the three grids which highlights the grid independency of the computed results.

For the fluid dynamics part of the problem, a uniform velocity based on the flow Reynolds number was defined at the inlet $(\vec{V}=U=$ constant $)$ of the tube. No slip condition $(\partial \vec{V} / \partial r=0)$ was applied at the solid, nonporous, and stationary wall of the tube and zero gage pressure (i.e., $p_{\text {out }}$ $\left.=p_{\text {atm }}\right)$ was applied at the outlet. For the heat transfer part, at the inlet, a uniform temperature $\left(T=105^{\circ} \mathrm{C}\right)$ was defined. At the wall a convection condition $\left(q_{\text {conv }}^{\prime \prime}=h_{\infty}\left(T_{w}-T_{\infty}\right)\right)$, where $q_{\text {conv }}^{\prime \prime}$ is the convective heat flux and $T_{w}$ is the temperature of the wall, was assumed with a fixed heat transfer coefficient of $h_{\infty}=10 \mathrm{~W} / \mathrm{m}^{2} \mathrm{~K}$ for still air and an ambient air temperature of $T_{\infty}=25^{\circ} \mathrm{C}$. At the outlet, convective flow was assumed which mathematically states zero temperature gradient at the exit boundary $(\partial T / \partial z=0)$. The initial condition for the fluid flow in the entire domain was set to a uniform velocity that was equal to the inlet velocity and zero gage pressure, while for the heat transfer it was uniform temperature equal to the inlet temperature.

\section{Results and Discussions}

Figure 4 indicates that at any given value of $\lambda$ and $a$ the increase in the heat output with the Re is not significant; therefore, at particular ambient conditions the wave pitch $\lambda$ and amplitude $a$ are the only important parameters that affect the heat output from the wavy tube. Consequently, Figure 5 shows that the normalized heat output increases if $\lambda$ decreases and $a$ increases. Decreasing the pitch and increasing the amplitude increase the effective length of the tube which means increase in the overall heat transfer area. This increase 


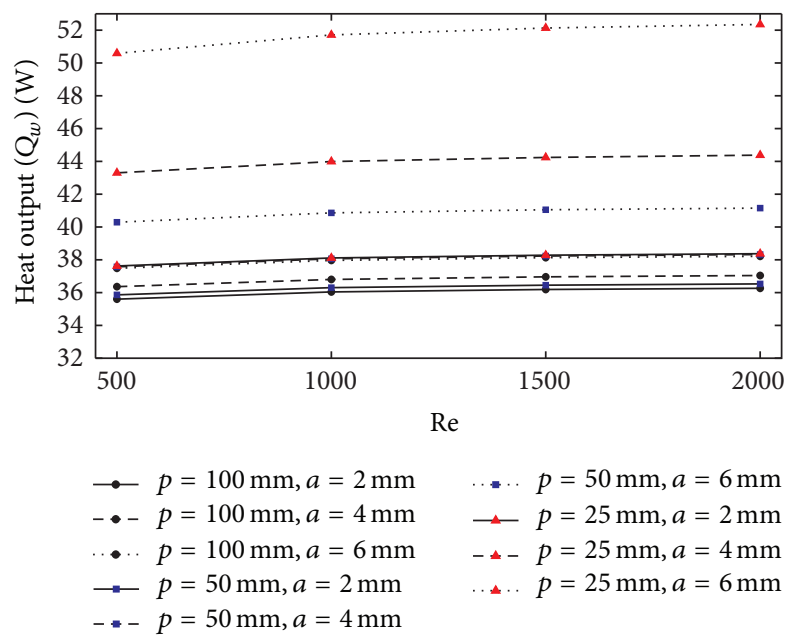

FIGURE 4: Heat output from the wavy tube as a function of $\operatorname{Re}, \lambda$, and $a$.

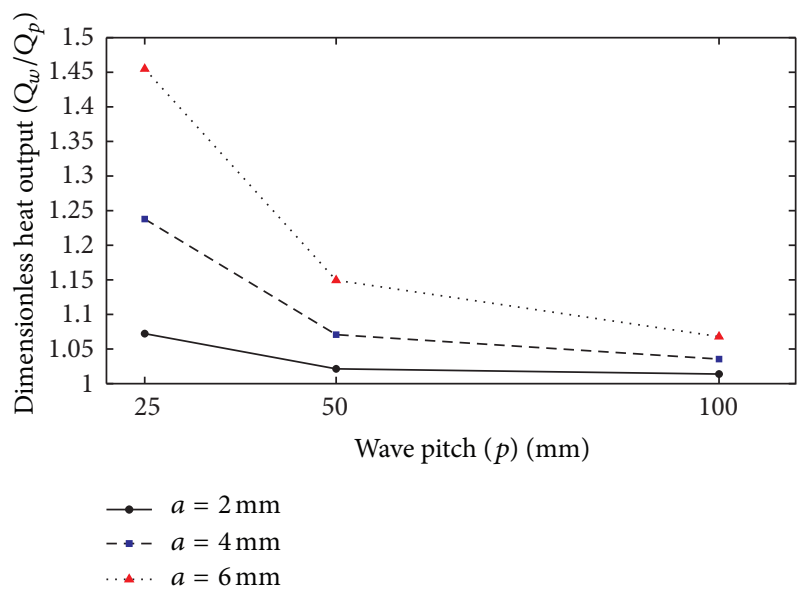

FIGURE 5: Normalized heat output from the wavy tube as a function of $\lambda$ and $a$.

in the heat transfer area is the main cause of the enhanced heat output from the wavy tube. For the case of $\lambda=25 \mathrm{~mm}$ and $a=6 \mathrm{~mm}$ the heat transfer area increases by $48 \%$ which causes an increase of approximately $46 \%$ in the heat output as compared to the plane circular tube of same dimensions (see Figure 5). As an example of space heating, 50 wavy-peristaltic tubes having $\lambda=25 \mathrm{~mm}$ and $a=6 \mathrm{~mm}$ combined together in form of a laminar convector can increase the temperature of air inside $10 \times 10 \times 10 \mathrm{~m}^{3}$ room from $10^{\circ} \mathrm{C}$ to $22^{\circ} \mathrm{C}$ in just 60 minutes. On the other hand decreasing $\lambda$ and increasing $a$ cause the pressure drop across the tube to increase. This effect is shown in Figure 6. From Figure 6 it can also be seen that, unlike the heat output, this time Re also has significant effect on the pressure drop. Figure 7 represents the typical streamline patterns within the diverging or bulge part of the wavy tube. The streamline pattern confirms the presence of circulation within the diverging part of the wavy tube. It can be observed that the size of the circulation zone increases as the wave amplitude $a$ increases. The presence of circulation

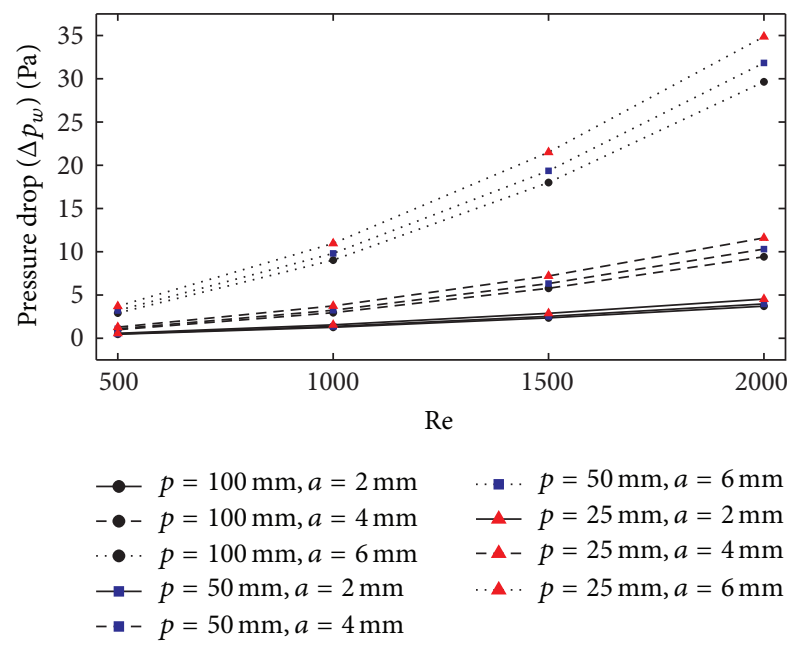

Figure 6: Pressure drop across the wavy tube as a function of $\operatorname{Re}, \lambda$, and $a$.

also indicates adverse pressure gradient effects which is also the reason of high overall pressure drop for the wavy tube. It is worth mentioning that streamline patterns indicating circulation within the diverging parts of the wavy tube were also identified in the works of Kim et al. [10] and Yang et al. [9].

It is to be noted that high thermal conductivity of liquid sodium is capable of counterbalancing the low convective effects of a laminar flow; therefore, it is possible to operate wavy tubes at low Re in order to reduce the pressure drop across the tube and at the same time increase the heat output by reducing $\lambda$ and increasing $a$. For instance, at $\lambda=25 \mathrm{~mm}$ and $a=6 \mathrm{~mm}$, the pressure drop across the tube at $\mathrm{Re}=2000$ is $35 \mathrm{~Pa}$; however, if $\mathrm{Re}$ is reduced to 500 the pressure drop reduces to $3.7 \mathrm{~Pa}$ (i.e., $89 \%$ reduction in the pressure drop) with only $3.4 \%$ reduction in the heat output of the tube. From Figure 6 it can also be noticed that compared to the wave pitch $\lambda$ the wave amplitude $a$ has a more prominent effect on the pressure drop.

Based on the above results, it can be concluded that Re and $a$ are the controlling parameters for optimizing the pressure drop across the wavy tube, whereas the heat output can be optimized by controlling $\lambda$ and $a$ independent of Re.

Since, from Figure 5, it is evident that the heat output increases if $\lambda$ decreases and $a$ increases, further simulations were performed to study the effect of heat transfer coefficient $h_{\infty}$ and the temperature of the ambient air $T_{\infty}$ on the exit temperature of sodium at $\operatorname{Re}=500, \lambda=25 \mathrm{~mm}$, and $a=$ $6 \mathrm{~mm}$. It was found that with an inlet temperature of $105^{\circ} \mathrm{C}$ the exit temperature of liquid sodium always remains above its melting point of $97.8^{\circ} \mathrm{C}$ for all values of $h_{\infty} \leq 10 \mathrm{~W} / \mathrm{m}^{2} \mathrm{~K}$ and $T_{\infty} \geq 0^{\circ} \mathrm{C}$. Therefore, Figure 8 depicts the variation of exit sodium temperature and the heat output as a function of $T_{\infty}$. From Figure 8 it can be concluded that the exit temperature of sodium remains higher and almost constant with the variation in $T_{\infty}$ whereas the heat output decreases as $T_{\infty}$ increases; however, this decrease is only less than $10 \%$ and 


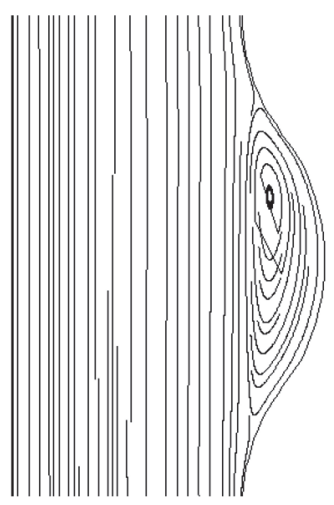

(a)

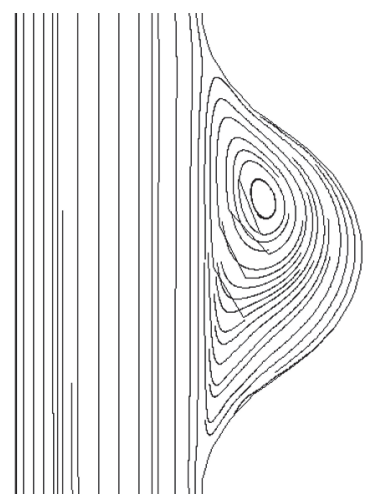

(b)

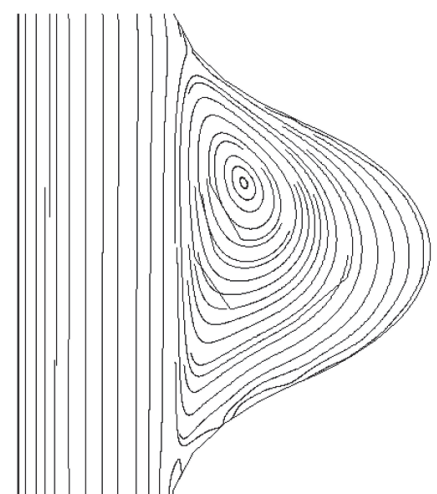

(c)

FIGURE 7: Typical streamline pattern within the diverging part of the wavy tube (a) at $\lambda=25 \mathrm{~mm}$ and $a=2 \mathrm{~mm}$, (b) at $\lambda=25 \mathrm{~mm}$ and $a=4 \mathrm{~mm}$, and (c) at $\lambda=25 \mathrm{~mm}$ and $a=6 \mathrm{~mm}$.

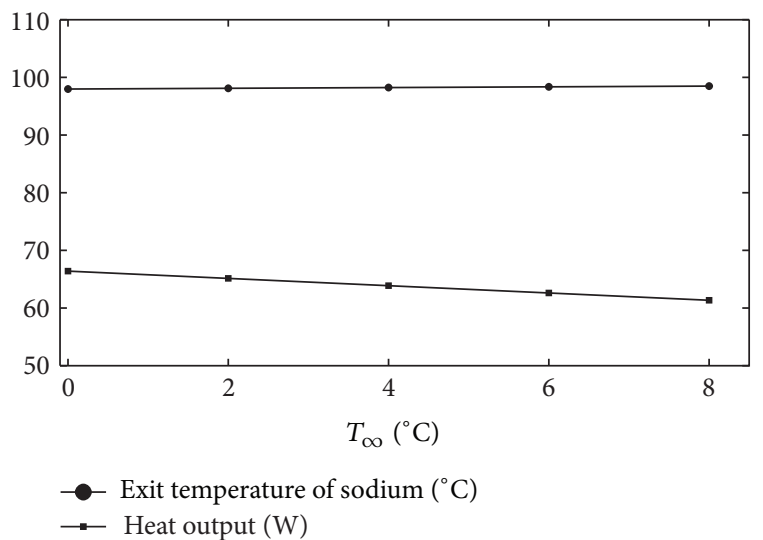

FIGURE 8: Variation of exit sodium temperature and heat output as a function of ambient air temperature at $h_{\infty}=10 \mathrm{~W} / \mathrm{m}^{2} \mathrm{~K}, \operatorname{Re}=500$, $\lambda=25 \mathrm{~mm}$, and $a=6 \mathrm{~mm}$.

therefore remains insignificant for the type of thermal system studied in this work.

\section{Conclusions}

In summary, effect of Reynolds number Re, wave amplitude $a$, and wave pitch $\lambda$ is studied numerically for flow of liquid sodium under laminar developing flow regime inside a wavy tube. It was found that the Reynolds number has insignificant effect on the heat output and can be ignored making the wave pitch and wave amplitude the controlling parameters for the optimization of the heat output. On the other hand, the pressure drop across the wavy tube is mostly affected by the Reynolds number and the wave amplitude. It is demonstrated that the wavy tube under the studied flow regime can be used as efficient heat transfer elements in laminar convectors for domestic and aerospace heating applications.

\section{Competing Interests}

The authors declare that they have no competing interests.

\section{Acknowledgments}

The authors would like to thank the governing authorities at the NED University of Engineering and Technology, Pakistan, King Abdulaziz University, Saudi Arabia, and Jeju National University, South Korea, for their sincere support.

\section{References}

[1] F. D. Doty, G. Hosford, J. D. Jones, and J. B. Spitzmesser, "A laminar-flow heat exchanger," in Proceedings of the 25th Intersociety Energy Conversion Engineering Conference (IECEC '90), vol. 4, pp. 1-7, Reno, Nev, USA, August 1990.

[2] W. M. Kays, "Numerical solutions for laminar flow heat transfer in circular tubes," Transactions of the ASME Journal of Heat Transfer, vol. 77, pp. 1265-1272, 1955.

[3] T. H. Ko, "Analysis of optimal Reynolds number for developing laminar forced convection in double sine ducts based on entropy generation minimization principle," Energy Conversion and Management, vol. 47, no. 6, pp. 655-670, 2006.

[4] J. C. Chen and J. S. Chiou, "Laminar and turbulent heat transfer in the pipe entrance region for liquid metals," International Journal of Heat and Mass Transfer, vol. 24, pp. 1179-1189, 1981.

[5] A. Z. Sahin and B. R. Mansour, "Entropy generation in laminar fluid flow through a circular pipe," Entropy, vol. 5, no. 5, pp. 404416, 2003.

[6] S. M. Mehdi and K. H. Choi, "Heat transfer coefficient for liquid sodium in developing laminar flow regime," in Proceedings of the International Conference on Energy and Sustainability, vol. 1, pp. 157-160, Karachi, Pakistan, 2013.

[7] E. Gedik, H. Kurt, and Z. Recebli, "CFD simulation of magnetohydrodynamic flow of a liquid-metal galinstan fluid in circular pipes," Fluid Dynamics and Materials Processing, vol. 9, no. 1, pp. 23-33, 2013.

[8] O. D. Makinde and A. S. Eegunjobi, "Effects of convective heating on entropy generation rate in a channel with permeable walls," Entropy, vol. 15, no. 1, pp. 220-233, 2013.

[9] Y.-T. Yang, Y.-H. Wang, and P.-K. Tseng, "Numerical optimization of heat transfer enhancement in a wavy channel using nanofluids," International Communications in Heat and Mass Transfer, vol. 51, pp. 9-17, 2014. 
[10] Y. J. Kim, M. Kim, S. Kim, J. K. Min, and M. Y. Ha, "Numerical study of fluid flow and convective heat transfer characteristics in a sinusoidal wavy circular tube," Journal of Mechanical Science and Technology, vol. 30, no. 3, pp. 1185-1196, 2016.

[11] C. H. Stignor, Laminar-flow liquid-to-air heat exchangers energy-efficient display cabinet applications [Ph.D. thesis], Lund University, Lund, Sweden, 2009.

[12] A. Miner and U. Ghoshal, "Cooling of high-power-density microdevices using liquid metal coolants," Applied Physics Letters, vol. 85, no. 3, pp. 506-508, 2004.

[13] M. Tawk, Y. Avenas, A. Lebouc, and M. Petit, "Numerical study of a liquid metal mini-channel cooler for power semiconductor devices," in Proceedings of the 17th International Workshop on Thermal Investigations of ICs and Systems (THERMINIC '11), pp. 120-125, Paris, France, September 2011.

[14] W. K. Lin, K. C. Liaw, M. Z. Tsai, and M. G. Chu, "Heat transport study of the laminar heat pipe heat exchanger," Smart Grid and Renewable Energy, vol. 3, no. 4, pp. 348-354, 2012.

[15] M. Iqbal and K. S. Syed, "Analysis of thermally developing laminar convection in the finned double-pipe heat exchanger," Heat Transfer Research, vol. 45, no. 1, pp. 1-21, 2014.

[16] N. Saji, S. Nagai, K. Tsuchiya, H. Asakura, and M. Obata, "Development of a compact laminar flow heat exchanger with stainless steel micro-tubes," Physica C: Superconductivity, vol. 354, no. 1-4, pp. 148-151, 2001.

[17] P. Sagnik and S. K. Saha, "Laminar flow and heat transfer through a circular tube having integral transverse corrugations and fitted with centre-cleared twisted-tape," Experimental Thermal and Fluid Science, vol. 57, pp. 388-395, 2014.

[18] Y. Sui, C. J. Teo, P. S. Lee, Y. T. Chew, and C. Shu, "Fluid flow and heat transfer in wavy microchannels," International Journal of Heat and Mass Transfer, vol. 53, no. 13-14, pp. 2760-2772, 2010.

[19] N. E. Todreas and M. S. Kazimi, Nuclear Systems I: Thermal Hydraulic Fundamentals, Taylor \& Francis, New York, NY, USA, 2nd edition, 1993.

[20] V. Sobolev, "Database of thermophysical properties of liquid metal coolants for GEN-IV,' Scientific Report SCK.CEN-BLG1069, 2010.

[21] R. W. Lewis, P. Nithiarasu, and K. N. Seetharamu, Fundamentals of the Finite Element Method for Heat and Fluid Flow, John Wiley \& Sons, London, UK, 2003.

[22] COMSOL Multiphysics Version 3.5a, Reference Guide, 2008.

[23] M. Sahu, K. K. Khatua, K. C. Patra, and T. Naik, "Developed laminar flow in pipe using computational fluid dynamics," in Proceedings of the 7th International R\&D Conference on Development and Management of Water and Energy Resources, Bhubaneswar, India, February 2009. 


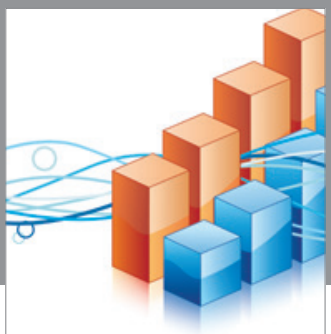

Advances in

Operations Research

vatem alat4

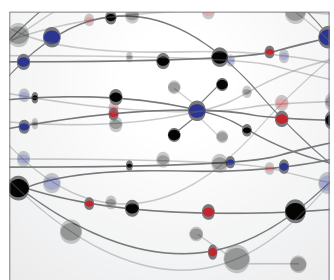

\section{The Scientific} World Journal
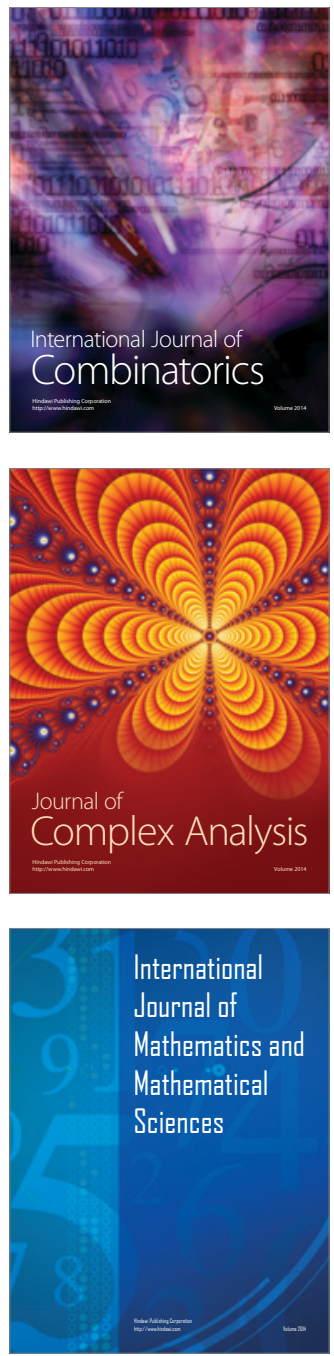
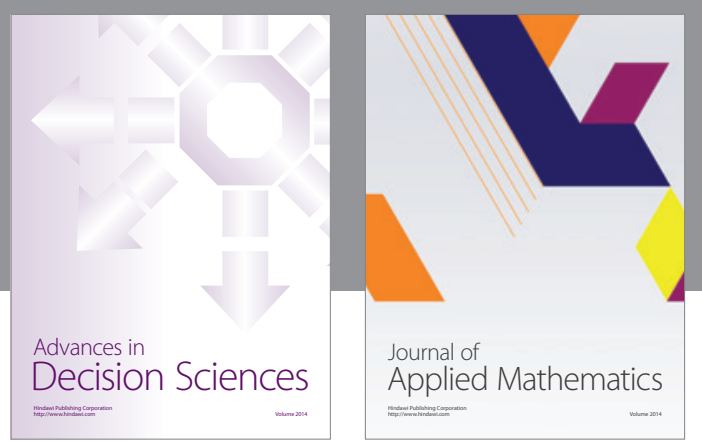

Algebra

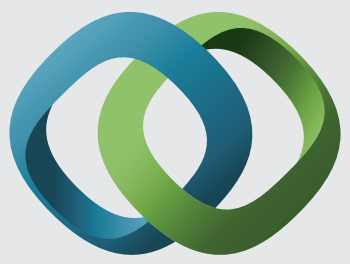

\section{Hindawi}

Submit your manuscripts at

http://www.hindawi.com
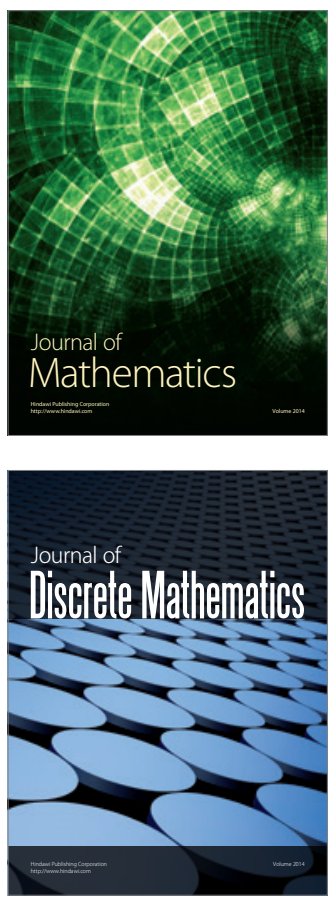

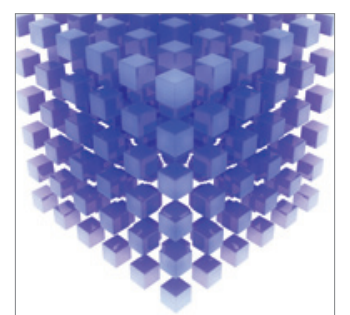

Mathematical Problems in Engineering
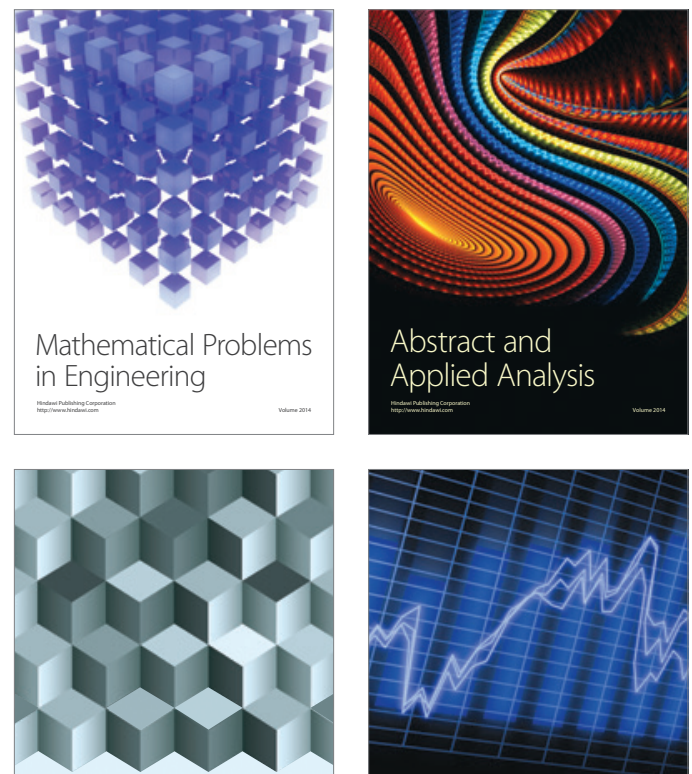

Journal of

Function Spaces

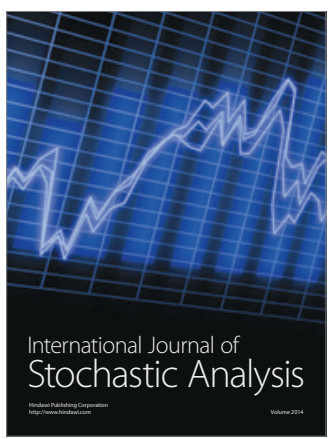

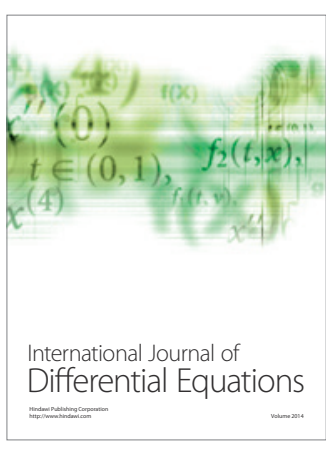
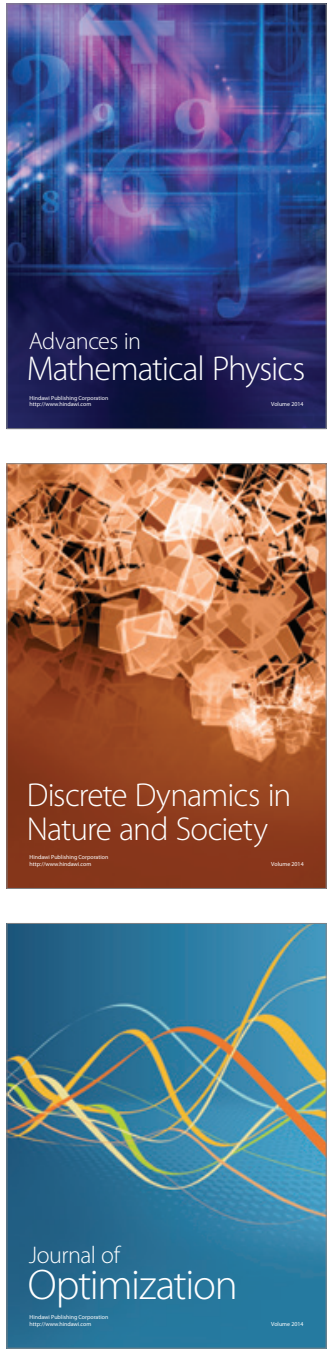